\title{
SOME NOTES ON MY LIFE AS AN ASTRONOMER
}

J. H. Oort

Sterrewacht Leiden, Leiden, The Netherlands

When in 1917, at the age of 17, I began my studies in Groningen I became almost immediately inspired by Kapteyn's lectures on elementary astronomy. Although I had been strongly interested in astronomy since my high school years in Leiden, and this had influenced my choice of the University of Groningen because Kapteyn was there, I was, in 1917, still undecided between physics and astronomy as my major direction. It was Kapteyn's influence that turned me definitely to astronomy; I remember that I was so impressed by the way he taught elementary celestial mechanics that I tried to convey my new insight to friends who had likewise just entered the University, but were studying humanities. But I do not believe that I succeeded in conferring to them a full appreciation of the fascination of celestial dynamics.

Perhaps the most significant thing I learned-mainly, I believe from Kapteyn's discussion of Kepler's method of studying nature-was to tie interpretations directly to observations, and to be extremely wary of hypotheses and speculations. In the first part of his course, Kapteyn refrained, for instance, from introducing the notion of "force" to replace the measurable quantity "acceleration." He disliked intricate mathematical formulations which prevented one from "seeing through" a theory; he feared the danger that the formulae might make one lose sight of the essentials. This was, of course, before quantum mechanics brought home the fact that one's sight is insufficiently developed to "look through" the deeper domains of physical science without the aid of mathematics.

At the time I studied in Groningen its Astronomical Laboratory was fully occupied by the first exploration of the Galaxy. It was the period, also, in which Shapley published his investigations of the globular clusters. A young student could not but wonder about the enigmatic contradiction between the picture produced by the star counts on one hand and that of the system of globular clusters on the other hand. It was clear enough that 


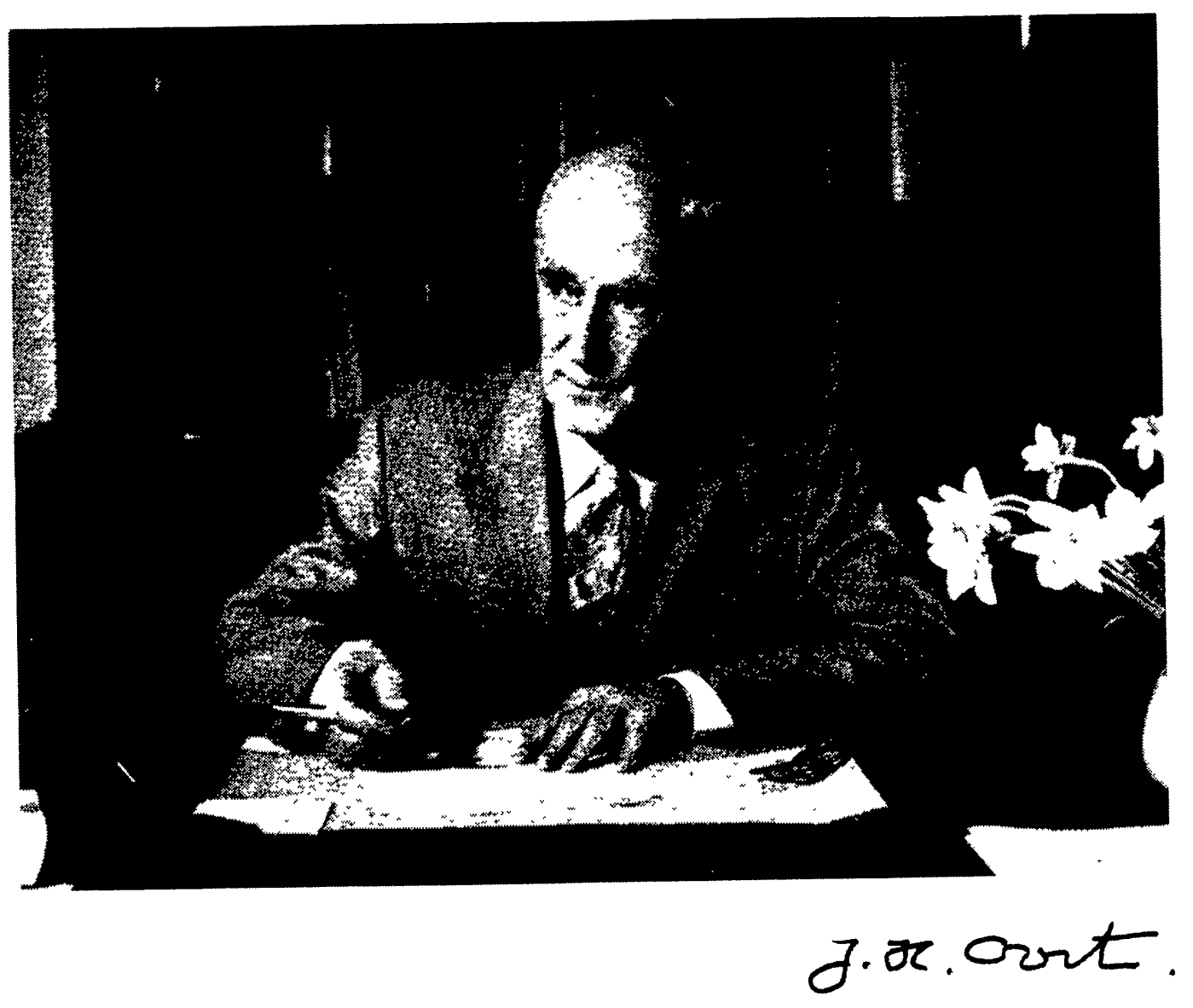

() Annual Reviews Inc. - Provided by the NASA Astrophysics Data System 
there must be a relation, because the center of the globular cluster system lay so precisely in the galactic plane, but what the relation was remained unclear until almost a decade later, when a bold step by Bertil Lindblad in the dynamics of a large galactic system indicated its true unified nature.

At the time, Shapley imagined a swarm of island systems, one of which was the Kapteyn System, the swarm extending over the large space occupied by the globular clusters. Kapteyn and van Rhijn, on the other hand, speculated at one time that the "cluster variables" used by Shapley to determine the distances of the clusters might be much fainter than assumed and the cluster system, therefore, much smaller, possibly of the dimension of the Kapteyn System. A remarkable feature noted in Shapley's work was that the globular clusters avoided a zone of about $8^{\circ}$ around the galactic equator. Shapley believed originally that this was due to a dissolution of the clusters passing through the central layer. I remember that I thought this quite impossible, and that the zone of avoidance must be due to obscuration. This contained the clue to the solution of the apparent conflict between the two galactic models. But li did not at the time draw the conclusion that the extent of the disk of Kapteyn's System might be limited by the same obscuration, and that in reality it might extend as far as the system of globular clusters. It took until 1925 for this correct picture to emerge.

Practically from the beginning of my study, I had been fascinated by the possibility that dynamics, which had so long been practically a monopoly of the astronomers investigating the solar system, might one day be applied to the so vastly greater systems of stars. An impressive foreshadowing was Kapteyn's "star streams" which Karl Schwarzschild subsequently interpreted as an ellipsoidal distribution of stellar motions.

A strong stimulation had come to me from reading a semi-popular book by Newcomb which Kapteyn had lent me. In later years there were the inspiring books by Eddington, Sitellar Movements and the Structure of the Universe, and by Jeans Problems off Cosmogony and Stellar Dynamics, both of which fundamentally influenced my education as an astronomer. The fascination of the dynamics of star clusters, stellar systems, galaxy clusters, and, finally, the still larger aggregates of galaxies called superclusters has remained with me to this day.

My first research in Groningen was concerned with high-velocity stars. My attention had been drawn to a puzzling observation by Adams and Joy. They had found that the radial-velocity vectors of a dozen stars with velocities of $150 \mathrm{~km} / \mathrm{s}$ and higher showed an outspoken one-sided distribution in galactic longitude. I wondered whether this peculiarity was a general property of high velocities, and proceeded to collect complete 
data on stars of somewhat lower velocity, but still sufficiently above the average to be exceptional. It turned out that there was a surprisingly sharp limit-around $65 \mathrm{~km} / \mathrm{s}$-above which the radial-velocity vectors were entirely confined to a semicircle, between about $150^{\circ}$ and $330^{\circ}$ longitude (on the old system), while the velocities lower than about $60 \mathrm{~km} / \mathrm{s}$ were evenly distributed over all longitudes. Following a suggestion by Kapteyn I hypothesized that the limit of $65 \mathrm{~km} / \mathrm{s}$ was related to the velocity of escape from the Kapteyn System, and that the high-velocity stars might be interlopers from outside this System.

The subject of the asymmetry of the high velocities was worked out in detail in my doctoral thesis of 1926 . But no really satisfactory explanation had yet been found.

No more than a year later, a revealing interpretation was given by Lindblad. He suggested that, rather than being an assembly of separate swarms of the nature of Kapteyn's System drifting in the large system of globular clusters, the real Galaxy would consist of a number of subsystems with various velocity dispersions, each of which had the same diameter as the globular cluster system. This picture led me to the discovery of the differential rotation of the Galaxy which gave a direct confirmation of Lindblad's model, and led him to a discussion of the random motions in a rotating stellar system, and to an explanation of the ellipsoidal velocity distribution, thus giving a solution of the problem of Kapteyn's "star streams" with which astronomers had been confronted since the beginning of the century.

And so dynamics of the Galaxy had become possible.

In fact, the first determination of galactic forces had already been made by Kapteyn five years earlier in his study of the distribution of stars perpendicular to the galactic plane. When trying to improve this work a decade later, with considerably more data, I found that the known stars and interstellar gas could account for only about two thirds of the dynamically derived value of $K_{z}$, and that there was, thus, an indication of a considerable unseen mass.

In the following half century, stellar dynamics lived through a tremendous development, in particular, by the undreamt possibilities opened by the advent of radio astronomy and the $21-\mathrm{cm}$ line. This enabled us to study the Galaxy's rotation and mass distribution down to its nucleus, and to determine its structure throughout the optically obscured disk. It revealed, besides, some quite unexpected things: the "three-kiloparsec arm" with its strongly noncircular motion, and also some even more striking structures closer to the center, with quite high velocity components directed away from the nucleus. 
Long before the appearance of radio astronomy, I had been strongly interested in the possibility of determining the field of force in elliptical galaxies and bulges of spirals in the hope that these might help us to understand spiral structure. So I applied in 1932 for observing time for such a program with the new 60-inch Perkins Observatory telescope in Delaware, Ohio. I wanted specifically to test Lindblad's first theory of spiral structure (which must, at least in part, have been inspired by Jeans's speculations based on the instability of McLaurin spheroids). I failed for two reasons: first, because of my almost total lack of observing experience; second, because the light distribution gave no indication of any instability region of the type required by Lindblad's theory. It was only many years later that at the McDonald Observatory, with the help of Hiltner in particular, the first successful measurements were obtained (Oosterhoff had meanwhile taken a number of excellent photometric plates for my program on Mt. Wilson).

It is only natural for an astronomer who got his first inspiration from Kapteyn's ideal to penetrate to the end of what was then the observable world to become profoundly fascinated by the prospects of penetrating to the end of the so much vaster world that became observable in the second half of this century. The new era abounded in wonderful discoveries, but the one that particularly stands out in my memory is the epic of the first successful descent into the past of the Universe, which was made by Ryle and his coworkers at the Cavendish Laboratory.

Radio astronomy clearly promised to become the tool par excellence for studying the Universe. Probably the greatest step was its leading to the discovery of quasars. In the quasars optical and radio astronomy were joining forces. One wonders what they will uncover in the coming ten or twenty years.

Sixty years of research comprised, of course, rather more than the few episodes I have mentioned. There was, for instance, the period of research during the war years, together with van de Hulst, on the formation, growth, and destruction of interstellar particles, a study that had resulted from the great need in that pre-radio period to obtain more insight into the nature of the formidable barrier of the absorption of light in the galactic disk. There were, much later, the high-velocity clouds, which promised to throw some light on how galaxies might continue to grow, long after their birth. There was also the episode of the Crab Nebula, which provided the exciting evidence that synchrotron radiation occurs in nature, an episode with which such exceptional friends as Duyvendak, 
Walraven, and Baade were so closely interwoven. Another interlude was devoted to comets and their history.

The frequent mentioning of Kapteyn in my early recollections might give a distorted picture of the personal influences that shaped my life as an astronomer. Actually, my contact with him was of short duration compared to the long period in Leiden with its almost daily extremely valuable contacts with Hertzsprung and de Sitter, and the many contacts by visits as well as correspondence with the astronomical world at large, the most fascinating of which were with Walter Baade.

Between Groningen and Leiden there had been two years in New Haven, where Frank Schlesinger taught me to measure the latitude variation with a photographic zenith telescope.

Curiously, the relations with de Sitter never touched the subject of the Universe, in which I became so strongly interested in later years. The Einstein-de Sitter Universe and all that pertained to the subject was hibernating at that time.

A Dutch historian recently wrote a book on her life called Omzien in Verwondering (Looking Back in Astonishment). My own life has been, and still is, one of marveling about what lies ahead. If I were to write a book on it, I would rather call it Looking Ahead in Wonder. 